\title{
The cosmic ray test of MRPCs for the BESIII ETOF upgrade
}

\author{
Xiaozhuang Wang ${ }^{1,2, a}$, Yuekun Heng ${ }^{2,3, b}$, Zhi $\mathbf{W u}^{2,3}$, Cheng $\mathbf{L i}^{1,2, c}$, Yongjie $\mathbf{S u n}^{1,2}$, Hongliang Dai ${ }^{2,3}$, \\ Shengsen Sun ${ }^{2,3}$, Rongxing Yang ${ }^{1,2}$, Zhen Liu ${ }^{1,2}$, Ping Cao ${ }^{1,2}$, Jie Zhang ${ }^{2,3}$, Yun Wang ${ }^{1,2}$, Weijia Sun ${ }^{1,2}$, \\ Siyu Wang ${ }^{1,2}$, Xiaolu $\mathbf{J i}^{2,3}$, Jinzhou Zhao ${ }^{2,3}$, Wenxuan Gong ${ }^{2,3}$, Mei $\mathbf{Y e}^{2,3}$, Xiaoyan $\mathbf{M a}^{2,3}$, Mingming Chen ${ }^{2,3}$, \\ Meihang Xü ${ }^{2,3}$, Xiaolan Luo ${ }^{2,3}$, Kejun $\mathbf{Z h u}^{2,3}$, Zhenan Liu ${ }^{2,3}$, Xiaoshan Jiang ${ }^{2,3}$ \\ ${ }^{1}$ Department of Modern Physics, University of Science and Technology of China, Hefei 230026, China \\ 2 State Key Laboratory of Particle Detection and Electronics, Beijing 100049, China \\ ${ }^{3}$ Institute of High Energy Physics, Chinese Academy of Sciences, Beijing 100049, China
}

Received: 1 December 2015 / Accepted: 8 April 2016 / Published online: 16 April 2016

(C) The Author(s) 2016. This article is published with open access at Springerlink.com

\begin{abstract}
In order to improve the particle identification capability of endcap time-of-flight (ETOF) at Beijing Spectrometer III, it is proposed to upgrade current ETOF detector with multi-gap resistive plate chamber (MRPC) technology aiming at extending ETOF overall time resolution to be $80-100$ ps for $\pi$ 's. The new ETOF system including MRPC detectors, front end electronics, CLOCK module, fast control boards and time-to-digital conversion modules, was built up and underwent 3-month cosmic ray test. The main purposes of the cosmic ray test are examining the construction quality of the system, testing the joint operation of all instruments and guaranteeing the system performance. The results show that MRPC time resolution is better than $80 \mathrm{ps,} \mathrm{average} \mathrm{effi-}$ ciency is about $98.5 \%$ and the noise rate of strip is $0.2 \mathrm{~Hz} / \mathrm{cm}^{2}$ at the threshold range for normal operation. These results indicate that the entire ETOF system would work well and satisfy the requirements of upgrade.
\end{abstract}

\section{Introduction}

The original endcap time-of-flight (ETOF) particle identification (PID) system of the Beijing Spectrometer III (BESIII) $[1,2]$ consists of two disks of 48 pieces of plastic scintillating counters covering the polar angle region of $0.83<|\cos \theta|<$ 0.96 as shown in Fig. 1. It consists of fast scintillators blocks

\footnotetext{
a e-mail: xzwang@ihep.ac.cn

b e-mail: hengyk@ihep.ac.cn

c e-mail: licheng@ustc.edu.cn
}

(BC204) readout by fine-mesh photomultiplier tubes (Hamamatsu R5924) [3]. The time resolution measured by ETOF detector is 138 ps for $\pi$ 's [4,5], which cannot completely satisfy the higher precision requirement of physics. One significant reason that can worsen the ETOF resolution is the multiple scattering of particles in the thick aluminium endcaps of the main drift chamber. The large numbers of secondary particles from FEE boards and cables between main drift chamber (MDC) and ETOF cause high multi-hits rate in a readout channel of the ETOF made of coarsely segmented scintillator blocks [6]. These multi-hits events distort both shape and amplitude of the output signals deteriorating the time resolution of ETOF and imposing more constraints for offline calibration and physics analysis. Another important reason is the uncertainties of the particle positions hitting the scintillators that produce jittering for the time resolution in the process of position-time correction.

The multi-gap resistive plate chamber (MRPC) [7] is a new type of gaseous detector, with excellent time resolution, high detection efficiency, relatively low cost and is insensitivity to neutral particles. MRPCs have been successfully used as TOF detectors in several experiments, such as ALICE at LHC [8, 9], STAR at RHIC [10,11]. The segmentation of the MRPC can be made sufficiently fine to suppress multi-hits events effectively at BESIII as discussed in Ref. [6]. The results of the full simulation [12] show that $K / \pi$ identification at $95 \%$ confidence level up to $1.4 \mathrm{GeV} / \mathrm{c}$ can be achieved. In 2012 the proposal [13] was approved to upgrade the current BESIII ETOF with the MRPC technology.

In the following sections, we will describe the ETOF upgrade system, including MRPC detectors, the readout electronics, the high voltage system, the gas system, and data acquisition system. The cosmic ray test of the entire ETOF system and relevant analysis results will be discussed. 


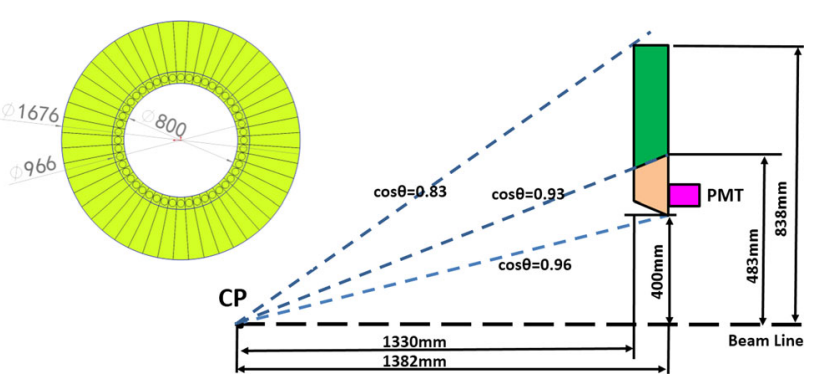

Fig. 1 Schematic drawing of ETOF location in BESIII spectrometer

\section{ETOF upgrade project}

\subsection{Design of BESIII ETOF}

There are 72 total MRPCs in the upgraded ETOF system and 36 on each end [13] as shown in Fig. 2. Adjacent modules are staggered slightly to avoid dead regions. The effective areas of the MRPC rings have inner radius of $501 \mathrm{~mm}$ and outer radius $822 \mathrm{~mm}$. Each MRPC is divided into 12 readout strips. The readout granularity is increased by 12 fold in $\mathrm{R}$ direction compared to the original ETOF in which each plastic scintillator module is readout by only one PMT from the inner end which will greatly decrease the position related timing jitter.

\subsection{Structure of ETOF MRPC}

The lengths of readout strips in each MRPC are from 9.1 $\mathrm{cm}$ to $14.1 \mathrm{~cm}$ and their width is $2.4 \mathrm{~cm}$ and separated by $3 \mathrm{~mm}$, as shown in Fig. 3. Strips are readout from both ends is used for each strip in order to improve the timing resolution and to help for determining the hit positions. The design of the MRPCs is optimized based on beam test for prototypes

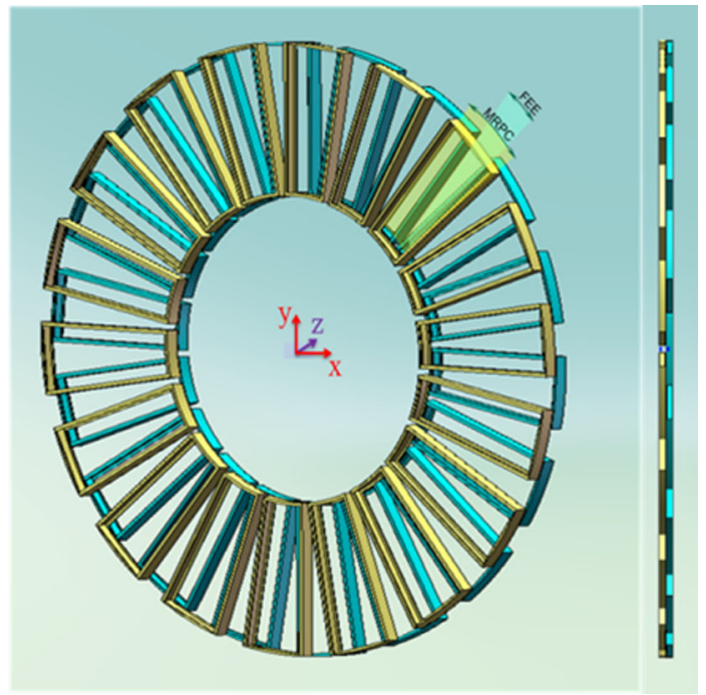

Fig. 2 The schematic drawing of MRPC ETOF upgrade at BESIII

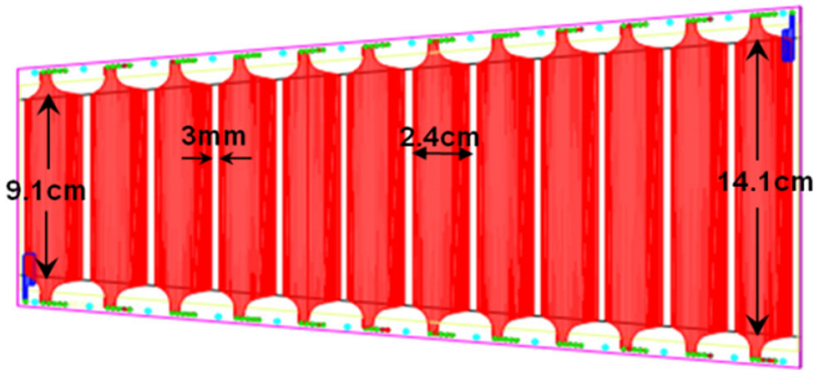

Fig. 3 The layouts of Printed Circuit Board

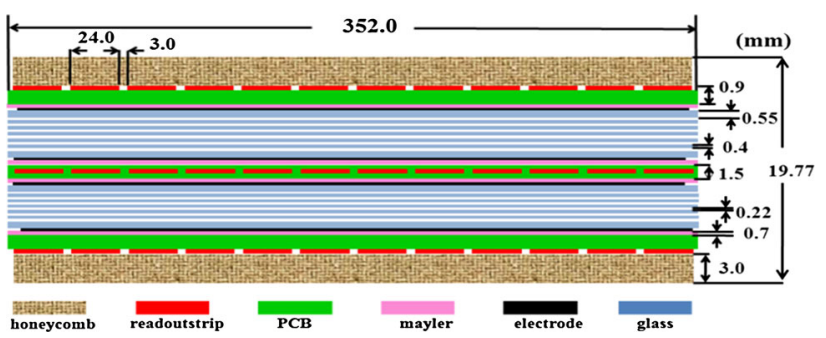

Fig. 4 The cross-sectional view of a MRPC module along it length

in which the single end and dual-end readout schemes are compared $[14,15]$.

Figure 4 shows the details of MRPC internal structure. Fourteen pieces of thin glass sheets are arranged into two stacks, and a stack is sandwiched in between two layers of readout strips. The middle layer readout strips are shared by the two stacks. There are total 12 active gas gaps and each gap is $0.22 \mathrm{~mm}$ thick that is defined by nylon fishing lines. The thicknesses of glass sheets are 0.4 and $0.55 \mathrm{~mm}$ for the inner and outer sheets respectively and their volume resistivity is $10^{13} \Omega \cdot \mathrm{cm}$. Graphite spray layer serving as high voltage electrodes are placed on the outer surfaces of the outermost glass sheets. The surface resistivity of the graphite spray layer is $\sim 5 \mathrm{M} \Omega / \square$. Two pieces of $3 \mathrm{~mm}$ thick glass fiber enforced epoxy honeycomb-boards are attached to the outer surfaces of the detector to ensure the flatness of the structure. The MRPC module is placed in a gas-tight aluminium box with a total thickness of $2.5 \mathrm{~cm}$ and the box is flushed with a gas mixture of $90 \%$ Freon+5\% $\mathrm{SF}_{6}+5 \%$ iso- $\mathrm{C}_{4} \mathrm{H}_{10}$.

\subsection{Readout electronics and data acquisition system}

The readout electronics system of MRPC detectors consists of FEE boards, Time-to-Digital (TDIG) convertion modules, Calibration-Threshold-Test-Power (CTTP) board, fast control module and a CLOCK module in NIM crates that communicates with and controlled by data acquisition (DAQ) system. The schematic of the readout electronics system process for the ETOF upgrade is shown in Fig. 5.

The FEE features four NINO chips developed by the ALICE-TOF group [16]. There are twenty four differential 


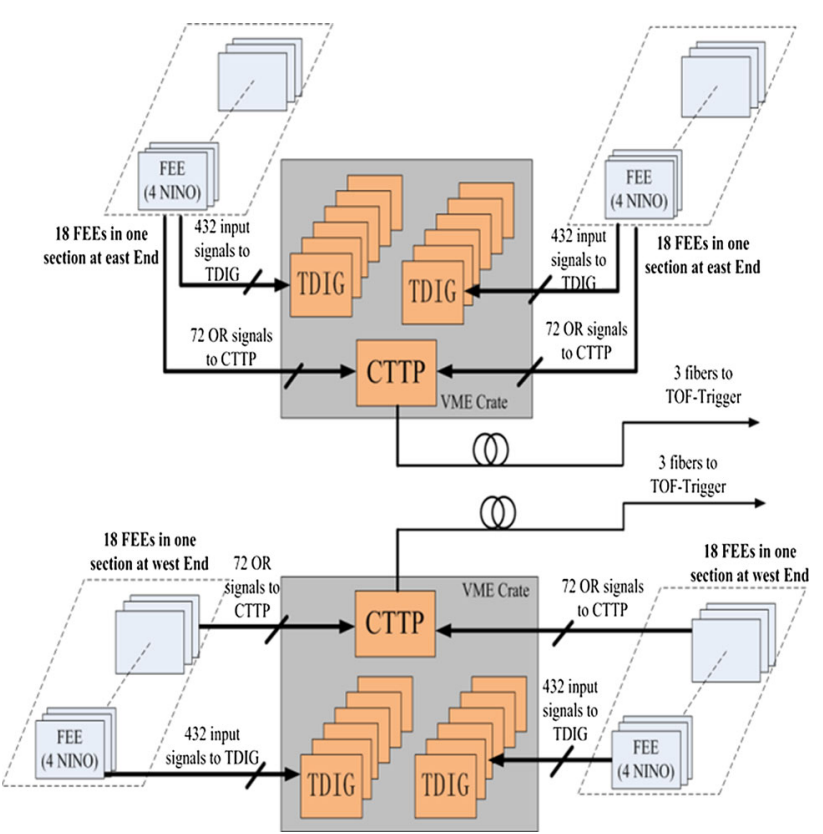

Fig. 5 The schematic of readout electronics system process

channels and correspondent LVDS signal, which convert signal charges into signals width. The timing accuracy is better than 15 ps RMS for each channel when the input charge is larger than $100 \mathrm{fC}$ [17]. The FEE boards with connectors and cables are fixed on the surfaces of the aluminium boxes of the MRPCs. A special designed short flexible printed circuit board connects the MRPC module outputs to the inputs of the NINO chips. For minimizing the time jitter and ensuring the signal quality, high-performance connectors (50 pin QSS-025-01-1-D-RA-MTI) and shielded differential cables (SQCD-025) from SAMTEC are used to connect the FEE and the TDIG [18].

The CTTP board housed in a NIM crate provides power, threshold and test signal for the FEE. It also receives the OR differential signals from the FEE and generates fast logic signals for TOF trigger subsystem.

The 9U VME crates consist of the VME controller of PowerPC board, readout control module of ROC, CLOCK module, and TDIG module. The TDIG modules are based on the HPTDC chips developed by the microelectronics group at CERN [19]. The principle of time measurements is demonstrated in Fig. 6. HPTDC measures the timing of both leading and trailing edges of the analog signals discriminated by the NINO chips with a pre-fixing threshold value. The time resolution of TDIG electronics system is approximately $25 \mathrm{ps}$ after integral non-linearity (INL) compensation [18]. After receiving and digitizing the signals in TDIG modules, data packing and uploading with predefined format are operated by the data acquisition (DAQ) system via the VME bus.

There are two working modes for the electronics system, data taking and calibration, which are determined by the ROC

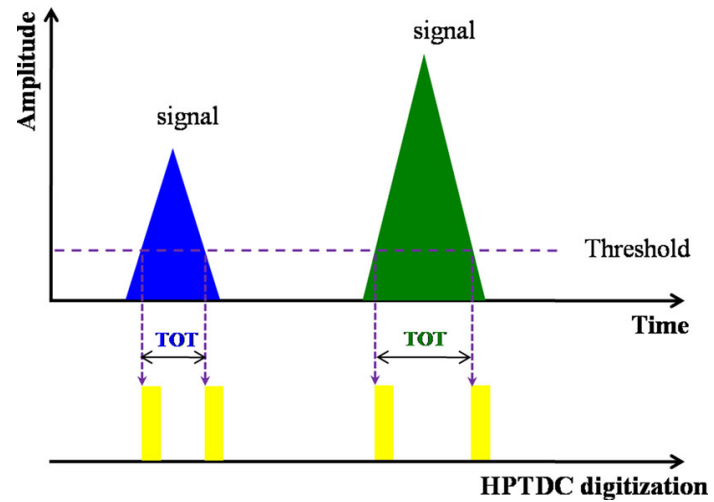

Fig. 6 The diagram of signals consequence in HPTDC digitization

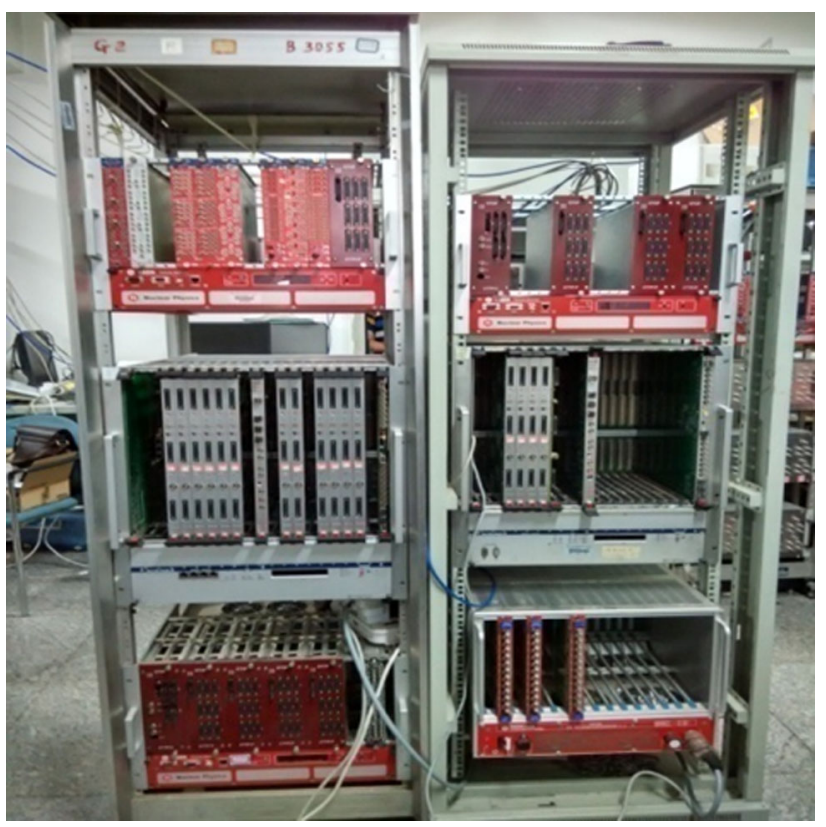

Fig. 7 The readout electronics system

module. At data taking mode of operation, the ROC receives a series of control signals, such as clock, trigger and sends them to the module of TDIG to start the system, while at the calibration mode the ROC generates these signals by itself to guarantee each channel's reliability of electronics system. The ROC module sends signals to CTTP to generate the test signals and to TDIG to control the time measurements. Upon the completion of measurements, the ROC module generates an interrupt signal on VME bus, and then data acquisition system will read out the data from the TDIG module and deliver them to DAQ computer.

The data acquisition system is similar to that at BESIII experiment, adopting the techniques of multi-level buffering, parallel processing, high-speed VME readout and network transmission. Also, the running status such as the event rate, noise level, the time spectrums are monitored by a computer program to provide a real-time information for the entire 


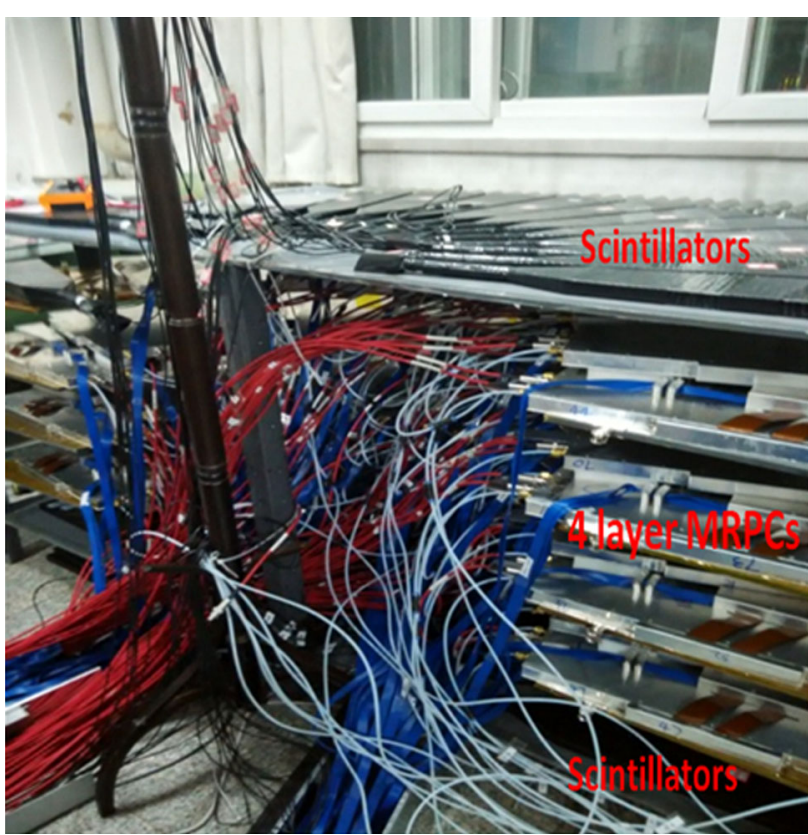

Fig. 8 The planting setup of MRPCs and scintillators

system. The picture of readout electronics system is shown in Fig. 7.

\subsection{Gas system and high voltage system}

The gas system consists of the gas supply bottles, mass flow controller, flow monitor and gas tanks. The composition of working gas mixture is $90 \%$ Freon $+5 \% \mathrm{~S} F_{6}+5 \%$ iso- $C_{4} H_{10}$ for the test. The gas components flow into a buffer tank with component ratio controlled by gas mass flow controller, and then delivered to MRPCs at a fixed rate of $400 \mathrm{ml} / \mathrm{min}$. In order to avoid the effect of moisture in MRPC, copper pipes are chosen to connect between gas tanks and MRPCs while

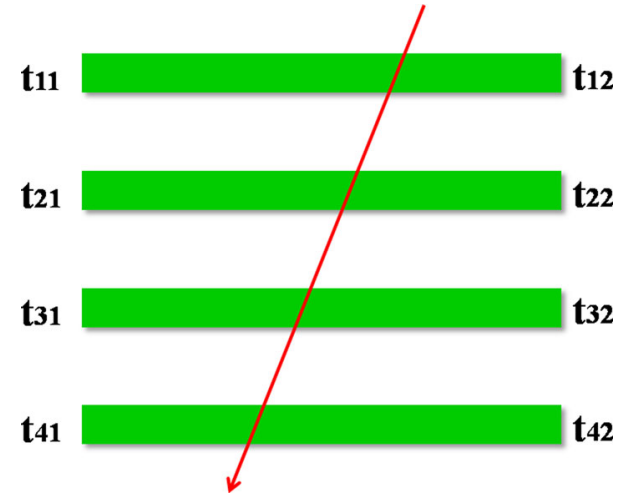

Fig. 10 The schematics of strips group hit by a cosmic ray

plastic pipes are used gas exhaust lines. Each three MRPCs are connected serially in a gas circuit.

The high voltage (HV) system, with nine pairs positive and negative channels for each endcap, consists of high voltage power supplies, HV distribution crate and cables. Each pair of channels are split in the HV distribution crate to supply four modules controlled by the BESIII slow control system.

\section{The Cosmic ray test}

\subsection{The system layout}

Three months cosmic ray test has been conducted in our laboratory. The MRPC working voltage is set at $\pm 7000 \mathrm{~V}$ and FEE threshold is set at $150 \mathrm{mV}$. MRPCs are stacked on four semicircle platforms as shown in Fig. 8. In order to trigger on the passing particles, 18 pairs of fan shaped scintillator counters, with active areas slightly smaller than the MRPC active area, are placed above and below the MRPC mod-

Fig. 9 The block diagram of system setup

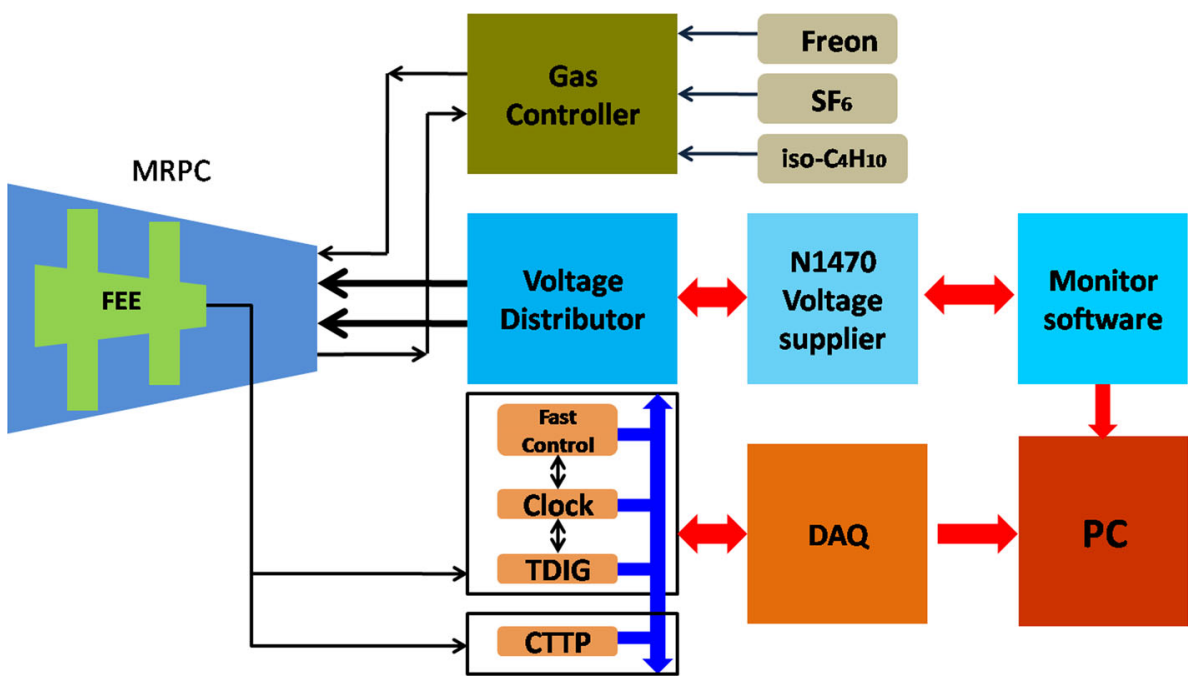




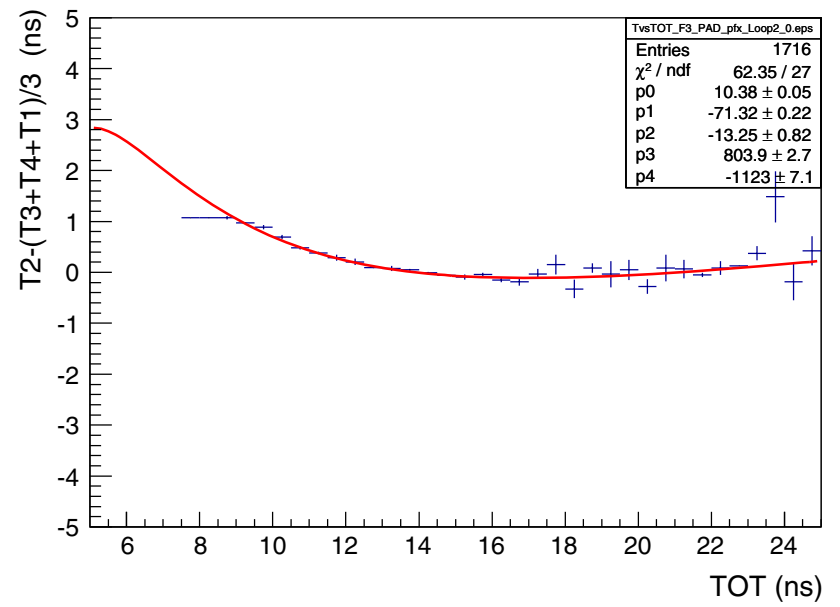

Fig. 11 The typical T-TOT fitted line with empirical function

ule separately. Figure 9 shows block diagram of the system setup.

\subsection{Data analysis}

For the convenience of data analysis, we select cosmic ray events passing through the same ID strips of the four MRPCs
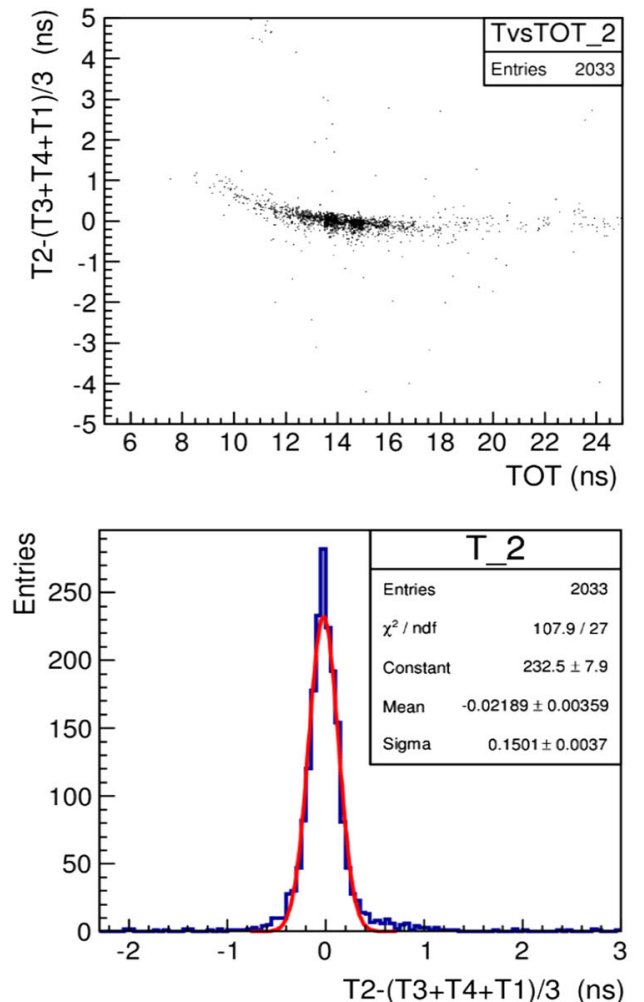

stacked vertically. Figure 10 shows the schematic of a cosmic ray event passing through them.

Two parameters are defined: $T_{i}$, and $T R_{i} . T_{i}$ represents mean time of strip; $T R_{i}=\left(T_{j}+T_{k}+T_{l}\right) / 3$ represents reference time which is independent of $T_{i}$. Four preliminary resolutions can be obtained from $\left(T_{i}-T R_{i}\right)$ distributions. The analysis process of cosmic ray test is briefly presented below:

Considering the time-amplitude effect, firstly fitting each scatter plot of $T_{i}-T R_{i}$ versus TOT $_{i}$ with empirical function as shown in Eq. (1):

$f(x)=p_{0}+\frac{p_{1}}{\sqrt{x}}+\frac{p_{2}}{x}+\frac{p_{3}}{x \sqrt{x}}+\frac{p_{4}}{x^{2}}$

Figure 11 shows the typical fitting line. Then using the corrected mean time $T_{j, k, l}($ new $)=T_{j, k, l}-f_{j, k, l}\left(\right.$ TOT $\left._{j, k, l}\right)$ to replace $T_{j, k, l}$ in $T R_{i}$ and repeat the process above. After several times iterations, the reference $T R_{i}$ is much accurate as shown in Fig. 12. After slewing correction four $T_{i}$ (new) $-T R_{i}$ (new) distributions can be obtained ultimately.

Define $\sigma_{i}^{\prime}(i=1,2,3,4)$ as measured resolution obtained by fitting the $T_{i}$ (new) $-T R_{i}$ (new) distribution with Gauss function as shown in Fig. 13, while $\sigma_{i}$ represents the time resolution of each MRPC strip. Eventually resolutions of four
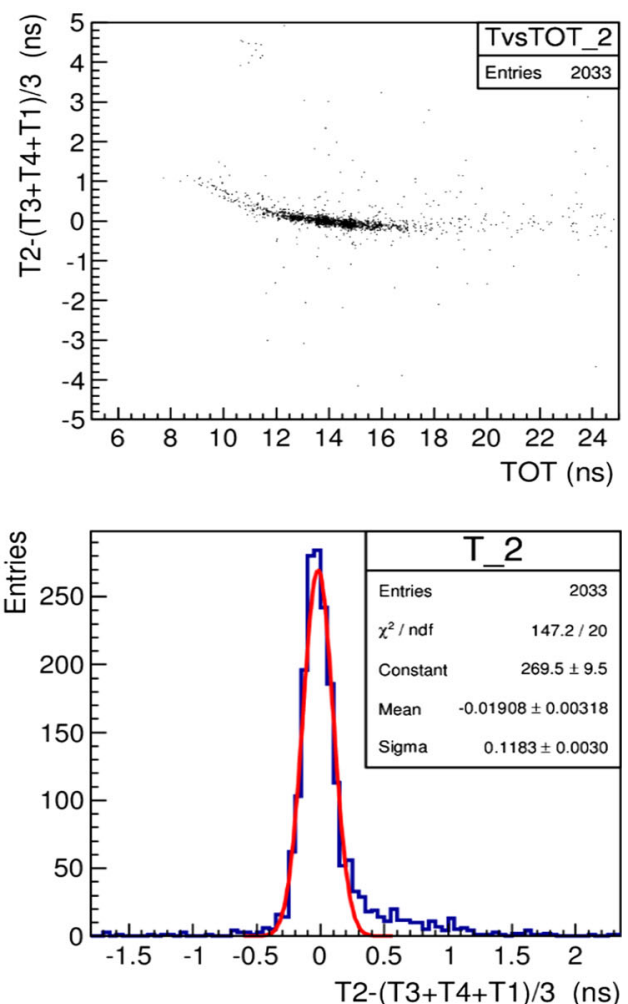

Fig. 12 Four figures before and after slewing correction: a scatter plot of $T_{2}-T R_{2}$ versus TOT 2 before iterations; $\mathbf{b}$ scatter plot of $T_{2}-T R_{2}$ (new) versus $\mathrm{TOT}_{2}$ after iterations; $\mathbf{c} T_{2}-T R_{2}$ distribution before iterations; $\mathbf{d} T_{2}-T R_{2}$ (new) distribution after iterations 


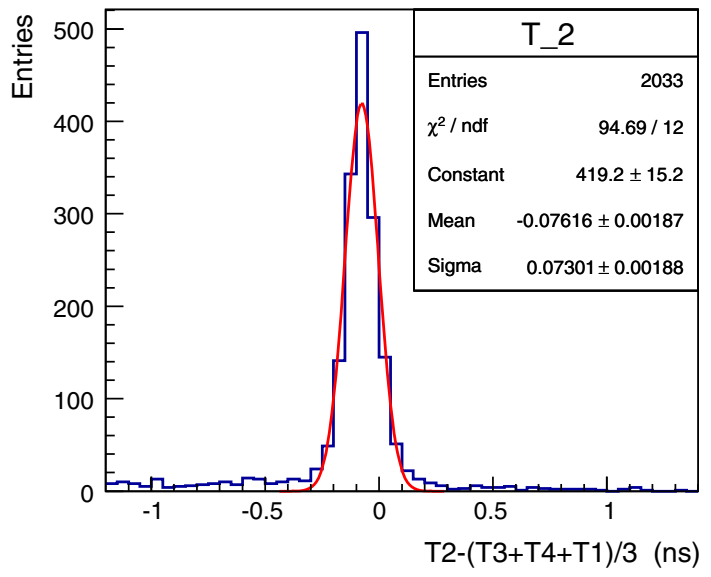

Fig. $13 T_{2}$ (new) $-T R_{2}$ (new) distribution after slewing correction

strips can be described in equations (2):

$\left\{\begin{array}{l}\sigma_{1}^{\prime 2}=\sigma_{1}^{2}+\left(\sigma_{2}^{2}+\sigma_{3}^{2}+\sigma_{4}^{2}\right) / 9 \\ \sigma_{2}^{\prime 2}=\sigma_{2}^{2}+\left(\sigma_{3}^{2}+\sigma_{4}^{2}+\sigma_{1}^{2}\right) / 9 \\ \sigma_{3}^{\prime 2}=\sigma_{3}^{2}+\left(\sigma_{4}^{2}+\sigma_{1}^{2}+\sigma_{2}^{2}\right) / 9 \\ \sigma_{4}^{\prime 2}=\sigma_{4}^{2}+\left(\sigma_{1}^{2}+\sigma_{2}^{2}+\sigma_{3}^{2}\right) / 9\end{array}\right.$

After solving this matrix equation, the time resolution of each strip can be obtained by the formula (3):

$$
\left(\begin{array}{c}
\sigma_{1}^{2} \\
\sigma_{2}^{2} \\
\sigma_{3}^{2} \\
\sigma_{4}^{2}
\end{array}\right)=\left(\begin{array}{cccc}
33 / 32 & -3 / 32 & -3 / 32 & -3 / 32 \\
-3 / 32 & 33 / 32 & -3 / 32 & -3 / 32 \\
-3 / 32 & -3 / 32 & 33 / 32 & -3 / 32 \\
-3 / 32 & -3 / 32 & -3 / 32 & 33 / 32
\end{array}\right)\left(\begin{array}{c}
\sigma_{1}^{\prime 2} \\
\sigma_{2}^{\prime 2} \\
\sigma_{3}^{\prime 2} \\
\sigma_{4}^{\prime 2}
\end{array}\right)
$$

This method is very useful for the Q\&A control in the mass production since it can get the test results of several MRPCs simultaneously.

\subsection{Test results}

By subtracting the $25 \mathrm{ps}$ jitter of electronics, the intrinsic resolution of MRPCs' strips can be obtained. The typical resolution results are shown in Fig. 14. The average intrinsic resolution of MRPC strips is approximately $60 \mathrm{ps}$. The resolutions of shortest and longest strip are a bit worse than middle strips, probably due to detector edge effect and low statistics. The uncertainty from momentum determination and flight time difference between strips of cosmic ray particles are also possible factors which deteriorate resolution slightly.

The detecting efficiency is determined by the ratio of number of hits in one strip to the number of tracks passing through the pad. As shown in Fig. 15, the average efficiency of strip is $98.5 \%$, which satisfies the design requirement of $\geq 96 \%$.

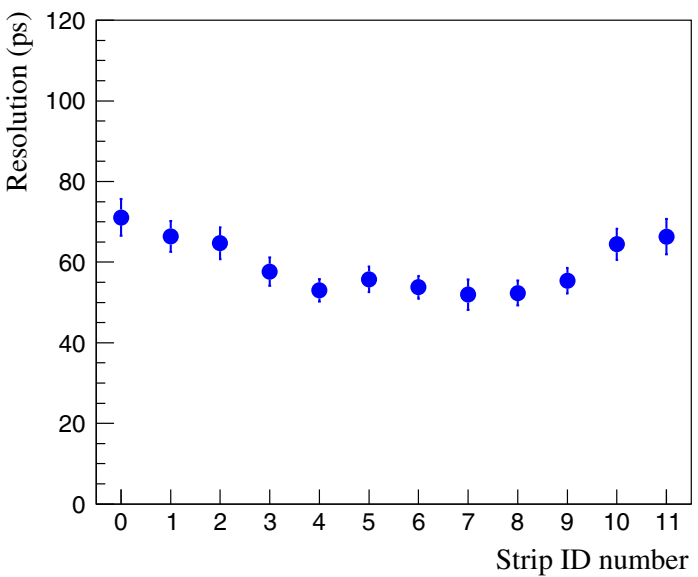

Fig. 14 The resolution versus strip ID

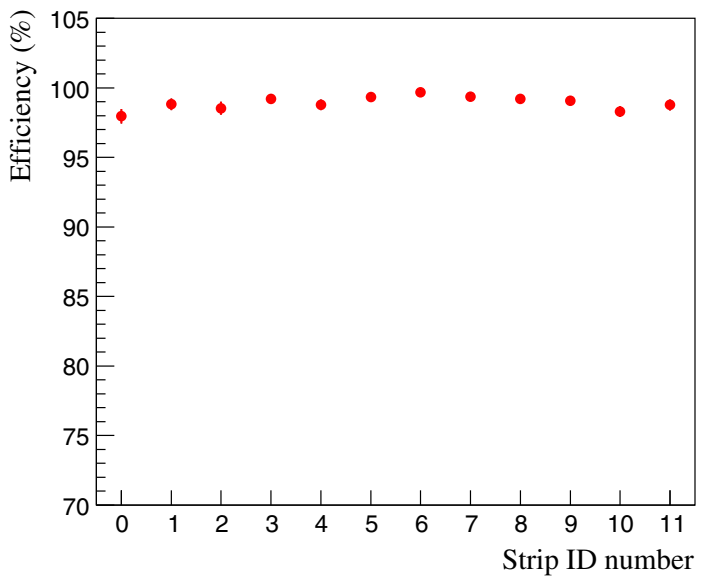

Fig. 15 The detecting efficiency versus strip ID

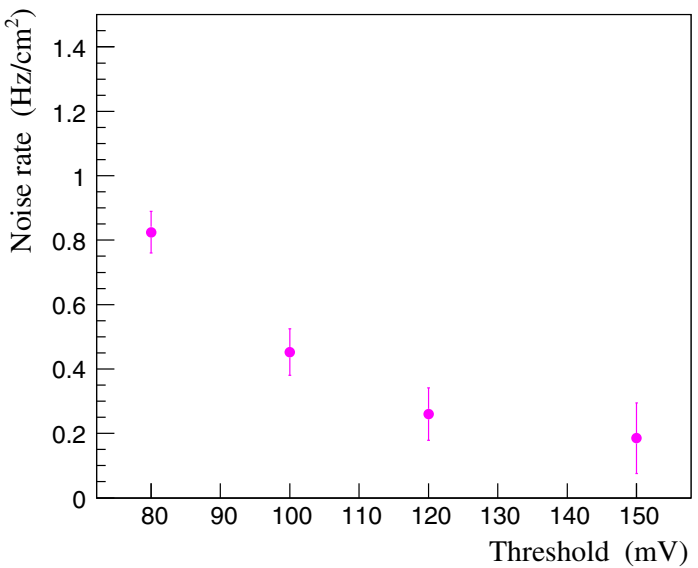

Fig. 16 The noise rate at different threshold

The noise rate of strips of every electronic channel is also studied. A signal independent of the cosmic ray was sent to the electronics as random trigger. As FEE threshold increases, the average noise rate of MRPC strips reduces and reaches a plateau at $0.2 \mathrm{~Hz} / \mathrm{cm}^{2}$ as shown in Fig. 16. The 
error bar of each point represents the noise rate fluctuations of different strips. It indicates that in normal working threshold range (usually more than $150 \mathrm{mV}$ ) the average noise rate is quite low.

\section{Conclusion}

The upgraded BES III ETOF system using the MRPC technology has been tested successfully. The intrinsic time resolution of MRPCs is measured to be approximately $60 \mathrm{ps,}$ while the high voltages of MRPCs are set to $\pm 7000 \mathrm{~V}$, significantly better than the intrinsic time resolution of the original ETOF using plastic scintillator blocks. The average detecting efficiency for cosmic ray muons is $98.5 \%$, and the average noise rate is approximately $0.2 \mathrm{~Hz} / \mathrm{cm}^{2}$ at the intended threshold range. These results prove that the performance of the MRPC based ETOF meets the requirements of the upgrade.

Acknowledgments This work is supported by the National Natural Science Foundation of China (No. 10979003) and Chinese Academy of Sciences (No. 1G201331231172010).

Open Access This article is distributed under the terms of the Creative Commons Attribution 4.0 International License (http://creativecomm ons.org/licenses/by/4.0/), which permits unrestricted use, distribution, and reproduction in any medium, provided you give appropriate credit to the original author(s) and the source, provide a link to the Creative Commons license, and indicate if changes were made. Funded by SCOAP ${ }^{3}$.

\section{References}

1. M. Ablikim et al., Design and construction of the BESIII detector, Nucl. Instrum. Meth. A, 614, 345-399 (2010)

2. BESIII Collaboration, The construction of the BESIII experiment. Nucl. Instrum. Meth. A 598, 7-11 (2009)
3. S.H. An et al., Testing the time resolution of the BESIII end-cap TOF detectors. Meas. Sci. Technol. 17, 2650-2654 (2006)

4. Z. Chuan et al., Time calibration for the end cap TOF system of BESIII. Chin. Phys. C. (HEP\&NP) 35(1), 72-78 (2011)

5. S.U.N. Shengsen, Time calibration for barrel TOF system of BESIII. J. Phys. Conf. Ser. 396, 022051 (2012)

6. Z. Hui, A GEANT4 simulation study of BESIII endcap TOF upgrade. Chin. Phys. C 37(9), 096002 (2013)

7. L. Cheng, A prototype of high time resolution MRPC. High Ener. Phys. Nucl. Phys. 25(9), 933-936 (2001)

8. D. De Gruttola et al., Study of the cosmic data taken with the ALICE TOF detector at the LHC. Nucl. Instrum. Meth. A 661, S102-S105 (2012)

9. A. Alici, The MRPC-based ALICE time-of-flight detector: status andperformance. Nucl. Instrum. Meth. A 706, 29-32 (2013)

10. F. Geurts, M. Shao et al., Performance of the prototype MRPC detector for STAR. Nucl. Instrum. Meth. A 533, 60-64 (2004)

11. W.J. Llope, The large-area time-of-flight upgrade for STAR. Nucl. Instrum. Meth. B 241, 306-310 (2005)

12. Y.K. Heng, Research Progress of MRPC ETOF. In: Proceedings of BESIII Collaboration 2010 Spring Meeting, 3 June 2010

13. M. Ullrich et al., Simulation of the BESIII endcap time of flight upgrade. Nucl. Instrum. Meth. A 769, 32-36 (2015)

14. S.U.N. Yong-jie et al., A prototype MRPC beam test for the BESIII ETOF upgrade. CPC(HEP \& NP) 36(5), 429-433 (2012)

15. S. Yang et al., Test of high time resolution MRPC with different readout modes for the BESIII upgrade. Nucl. Instrum. Meth. A 763, 190-196 (2014)

16. F. Anghinolfi et al., NINO: an ultrafast low-power front-end amplifier discriminator for the time-of-flight detector in the ALICE experiment. Nucl. Instrum. Meth. A 533, 183-187 (2004)

17. M. Despeisse et al., Low-power amplifier-discriminators for high time resolution detection. IEEE T. Nucl. Sci. 56, 375 (2009)

18. H. Fan et al., A high-density time-to-digital converter prototype module for BES III end-cap TOF upgrade. IEEE T. Nucl. Sci. 60, 3563 (2013)

19. M. Mota et al., A flexible multi-channel high-resolution time-todigital converter ASIC. In: IEEE Nucl. Sci. Conf. R., Lyon, France, pp. 15-20 (2000) 